\title{
Medición del impacto en la rentabilidad dada la implementación de un sistema de gestión en seguridad y salud en el trabajo en la empresa americana de curtidos LTDA. \& CIA. S.C.A
}

\author{
Measurement of impact on the performance given the implementation of a management \\ system in health and safety at work in the american tanning company LTDA. \& CIA. S.C.A
}

\author{
Santiago Vásquez Artunduaga ${ }^{1}$, Juan Carlos Correa Ruiz ${ }^{2}$, Luis Eduardo Hincapié Palmezano ${ }^{3}$ \\ ${ }^{1,2,3}$ Facultad de Ingeniería Industrial, Universidad Tecnológica de Pereira, Pereira, Colombia. \\ svasqueza@utp. edu.co \\ lehincapiedutp.edu.co \\ jccr2382@hotmail.com
}

\begin{abstract}
Resumen- El presente artículo, resultado de la investigación titulada: MEDICION DEL IMPACTO EN LA RENTABILIDAD DADA LA IMPLEMENTACION DE UN SISTEMA DE GESTION EN SEGURIDAD Y SALUD EN EL TRABAJO EN LA EMPRESA AMERICANA DE CURTIDOS LTDA. \& CIA. S.C.A, desarrolla una metodología completa que permite identificar los aspectos que mayor generan costos por el pago de conceptos relacionados con la gestión de salud y seguridad en el trabajo en la empresa antes relacionada, dado que no cuentan con la implementación del Sistema de Gestión en Seguridad y Salud Ocupacional en la Norma OSHAS 1800:2007 hasta el año 2013, se pronostican estos costos al 2014, así como también se proyectan Los Estados Financieros de la empresa; posteriormente y a partir de una tasa de reducción ${ }^{1}$ aplicada a estos estados financieros atribuible a la implementación del Sistema de Gestión en Seguridad y Salud Ocupacional, recalculados estos estados financieros (Simulando la implementación del SG-SYSO ${ }^{2}$ ) para el año 2014, se comparan y se concluye en función de la rentabilidad de la empresa antes de la implementación del SG-SYSO y después de esta, así mismo se analiza en particular el comportamiento de los Gastos Operacionales (Pues en estos están los costos debidos al SGSYSO).
\end{abstract}

Palabras clave - Estados financieros, rentabilidad, Sistema de Gestión en Seguridad y Salud en el Trabajo OSHAS 18001

Abstract - This article, the result of a research project entitled : MEASUREMENT OF IMPACT ON THE PERFORMANCE GIVEN THE IMPLEMENTATION OF A MANAGEMENT SYSTEM IN HEALTH AND SAFETY AT WORK IN THE

\footnotetext{
${ }^{1}$ Esta tasa de reducción aplicada se basa en la tasa promedio de accidentalidad de los últimos 6 años, en la matriz de riesgos actualizada y en políticas de reducción de costos operacionales dada la implementación futura del SGSYSO.

${ }^{2}$ La sigla SG-SYSO significa Sistema de Gestión en Seguridad y Salud Ocupacional.
}

AMERICAN TANNING COMPANY LTDA. \& CIA. SCA develops a comprehensive methodology to identify the factors with the greatest generate costs for the payment of concepts related to the management of health and safety at work in the company before related, since they do not have the implementation of the System Safety Management and Occupational Health OHSAS 1800:2007 Standard until 2013, these costs are forecast for 2014 as well as projected Financial Statements of the company; later and from a rate reduction applied to these attributable to the implementation of the Safety Management System and Occupational Health financial statements , these financial statements recalculated ( Simulating the implementation of SG- SYSO ) 2014 , are compared and it is concluded depending on the profitability of the company before implementing the SG- SYSO and after this, also discusses in particular the behavior of Operating Expenditures ( As these costs are due to SG-SYSO ) .

Key Word- Financial statements, profitability Management System in Safety and Health at Work OSHAS 18001

\section{INTRODUCCIÓN}

En los últimos años las empresas de todos los sectores dados los requerimientos tanto de sus proveedores, clientes internos como externos y reglamentaciones de tipo legal, vienen implementando sistema de gestión de calidad, ambientales, de salud y seguridad en el trabajo, etc, pero pocas por no decir que ninguna de estas evalúan su impacto económico en la rentabilidad de la empresa, partiendo de análisis de costos operacionales, gastos evitables dada la implementación de algún sistema de gestión, entre otros aspectos de suma importancia que deberían ser medidos, así como su impacto empresarial, social y en particular económico.

En concordancia con lo antes descrito, los investigadores y autores de la presente artículo titulado: MEDICION DEL IMPACTO EN LA RENTABILIDAD DADA LA IMPLEMENTACION DE UN SISTEMA DE GESTION EN 
SEGURIDAD Y SALUD EN EL TRABAJO EN LA EMPRESA AMERICANA DE CURTIDOS LTDA. \& CIA. S.C.A, analizaran como se plasma en el titulo este impacto en la empresa referida.

\section{CONTENIDO}

\section{A. Estados Financieros [1]}

La situación de una empresa y los resultados obtenidos como consecuencia de las transacciones mercantiles efectuadas en cada ejercicio, se presentan por medio de los llamados "Estados Financieros", los que se formulan con datos que figuran en la contabilidad, para suministrar esta información a los interesados en el negocio.

Los Estados Financieros, por consiguiente, son los documentos que muestran, cuantitativamente, ya sea total o parcialmente, el origen y la aplicación de los recursos empleados para realizar un negocio o cumplir determinado objetivo, el resultado obtenido en la empresa, su desarrollo y la situación financiera que guarda el negocio.

\section{B. Rentabilidad sobre el Patrimonio Neto (ROE) [2] [3]}

El retorno sobre el patrimonio neto (ROE, por sus siglas en inglés) es uno de los dos factores básicos en determinar la tasa de crecimiento de las ganancias de una empresa. El segundo es la reinversión de las utilidades. En un mundo en el que las compañías primero se valúan en función de los beneficios futuros esperados, su análisis es importante.

Maximizar este ratio es el principal objetivo de cualquier firma ya que expresa el porcentaje de remuneración que puede ofrecerse a los capitales propios (representados por el patrimonio neto), mostrando el lucro que los accionistas están obteniendo por su inversión.

\section{2.3. Pareto [4]}

Es una representación gráfica de los datos obtenidos sobre un problema, que ayuda a identificar cuáles son los aspectos prioritarios que hay que tratar.

También se conoce como "Diagrama ABC" o "Diagrama 20-80".

$\mathrm{Su}$ fundamento parte de considerar que un pequeño porcentaje de las causas, el $20 \%$, producen la mayoría de los efectos, el $80 \%$. Se trataría pues de identificar ese pequeño porcentaje de causas "vitales" para actuar prioritariamente sobre él.

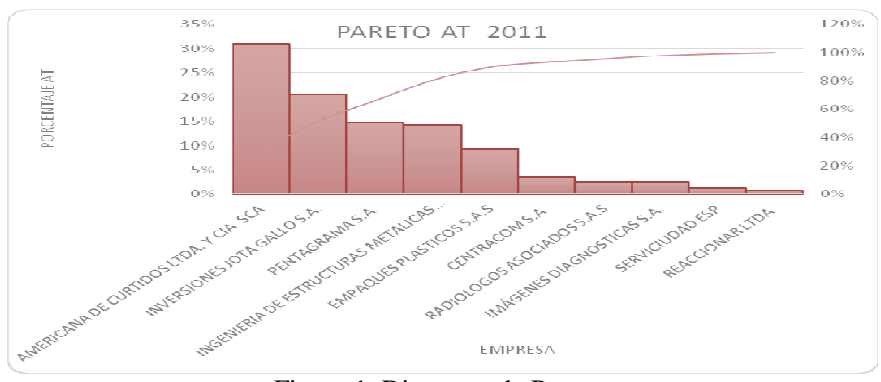

Figura 1. Diagrama de Pareto.

\section{Técnicas De Pronósticos [5]}

Los pronósticos son el primer paso dentro del proceso deplanificación de la producción y estos sirven como punto departida, no solo para la elaboración de los planes estratégicos, sino además, para el diseño de los planes a mediano y corto plazo, lo cual permite a las organizaciones, visualizar de maneraaproximada los acontecimientos futuros y eliminar en gran parte la incertidumbre y reaccionar con rapidez a las condiciones cambiantes con algún grado de precisión.

Predicciones:

Se basan meramente en la consideración deaspectos subjetivos dentro del proceso de estimación de eventosfuturos

Pronósticos:

Se desarrollan a través de procedimientoscientíficos, basados en datos históricos, que son procesadosmediante métodos cuantitativos.

PRONOSTICAR: Es emitir un enunciado sobre lo que es probable queocurra en el futuro, basándose en análisis y en consideraciones dejuicio. Se desarrollan a través de procedimientos científicos basados en datos históricos, que son procesados mediante métodoscuantitativos.

Pronósticos:

Hacer un pronóstico es obtener conocimiento sobre evento sin ciertos que son importantes en la toma de decisiones presentes.

Las técnicas depronósticosdisminuyen la incertidumbre sobre elfuturo, permitiendo estructurar planes y acciones congruentes conlos objetivos de la organización y permiten también tomar accionescorrectivas apropiadas y a tiempo cuando ocurren situaciones fuerade lo pronosticado. 


\section{TIPOS DE PRONOSTICOS}

\begin{tabular}{|l|l|}
\hline \multicolumn{1}{|c|}{ Nombre } & \multicolumn{1}{c|}{ Horizonte de predicción } \\
\hline No formales & Corto plazo \\
\hline Promedio simple & Corto plazo \\
\hline Promedio móvil & Corto plazo \\
\hline Suavización exponencial & Corto plazo \\
\hline Suavización exponencial lineal & Corto plazo \\
\hline Suavización exponencial cuadrítica & Corto plazo \\
\hline Suavización exponencial estacional & Corto plazo \\
\hline Filtración adaptiva & Corto plazo \\
\hline Descomposición clásica & Corto plazo \\
\hline Modelos de tendencia exponencial & Mediano y largo plazo \\
\hline Ajuste de curva S & Mediano y largo plazo \\
\hline Modelo de Gompertz & Mediano y largo plazo \\
\hline Curvas de crecimiento & Mediano y largo plazo \\
\hline Census II & Corto plazo \\
\hline Box-Jenkins & Corto plazo \\
\hline \multicolumn{1}{|c|}{ METODOS CUANTITATIVOS } \\
(TIPO SERIE DE TIEMPO)
\end{tabular}

Figura 2. Tipo de pronosticos.

E. Medidas de selección, precisión, e interpretación para métodos de pronósticos [6]

Para cada uno de los escenarios que usted genere es necesario medir el desempeño de ellos mediante indicadores de precisión. Se requiere definir un criterio para la precisión del pronóstico (datos de predicción) y otra para la selección del modelo (datos de control). No todos tendrán el mismo significado ni el mismo uso, pero ambos están basados en la siguiente fórmula del error (et):

$$
e_{t}=\left(Y_{t}-\hat{Y}_{t}\right)
$$

Donde et es el error del pronóstico,

Yt el valor observado o real en el tiempo $t$ de la serie de tiempo, y $\mathrm{Y}^{\wedge} \mathrm{t}$ es igual al valor pronosticado en el tiempo $t$ de la serie de tiempo.

El error también puede ser representado en términos relativos y/o absolutos (\%) utilizando la siguiente formulación:

$$
e a_{t}(\%)=\frac{\left|Y_{t}-\hat{Y}_{t}\right|}{Y_{t}} * 100
$$

$\mathrm{O}$ bien expresarlo en forma cuadrática:

$$
e_{t}^{2}=\left(Y_{t}-\hat{Y}_{t}\right)^{2}
$$

En la siguiente tabla se muestran las medidas de error más utilizadas donde $\mathrm{Ft}$ es igual a $\mathrm{Yt}$ notación utilizada para identificar el valor pronosticado.

Como se puede observar en dicha tabla existen más de 15 herramientas para medir el error del pronóstico, y su cantidad complica la selección de la medida de precisión más adecuada para el modelo de predicción. Por ejemplo, si seleccionamos aquellas de errores absolutos en lugar de los cuadráticos, éstos penalizan en mayor medida los errores grandes. La elección dependerá de la importancia que se les dé a los grandes errores. El coeficiente de desigualdad U de Theil, que no se muestra en la tabla y que se detalla más adelante, presenta una solución para estos escenarios. Si el valor de U es cercano a

\begin{tabular}{|c|c|}
\hline $\begin{array}{l}\text { Medida de error } \\
\text { MSE Mean Square Error }\end{array}$ & $\begin{array}{l}\text { Fórmula } \\
\text { Media\{ }\left\{e^{2}\right\}\end{array}$ \\
\hline RMSE Root Mean Square Error & $\sqrt{M S E}$ \\
\hline MAE Mean Absolute Error & Media \{e, [\} \\
\hline MdAE Median Absolute Error & Mediana\{e,|\} \\
\hline MAPE Mean Absolute Percentage Error & Media $\{p, \mid\}$ \\
\hline MdAPE Median Absolute Percentage Error & Mediana $\{p, \mid\}$ \\
\hline sMAPE Symmetric Mean Absolute Percentage Error & $\operatorname{Media}\left\{2 \cdot \frac{\left|Y_{r}-F_{i}\right|}{Y_{i}+F_{i}}\right\}$ \\
\hline sMdAPE Symmetric Median Absolute Percentage Error & Mediana $\left\{2 \cdot \frac{\left|Y_{t}-F_{r}\right|}{Y_{t}+F_{i}}\right\}$ \\
\hline MRAE Mean Relative Absolute Error & Media $\{r, \mid\}$ \\
\hline MdRAE Median Relative Absolute Error & Mediana $\left\{r_{r} \mid\right\}$ \\
\hline GMRAE Geometric Mean Relative Absolute Error & Media Glr, \\
\hline RelMAE Relative Mean Absolute Error & MAE $/$ MAE $^{*}$ \\
\hline RelRMSE Relative Root Mean Squared Error & RMSE / RMSE ${ }^{*}$ \\
\hline LMR Log Mean Squared Error Ratio & $\log ($ ReIRMSE $)$ \\
\hline PB Percentage Better & 100-Media $\left\{I\left\{r_{;}<1\right\}\right\}$ \\
\hline PB(MAE) Percentage Better (MAE) & 100-Media $\{$ \{ $\{$ MAE $<M A E *\}$ \\
\hline PB(MSE) Percentage Better (MSE) & 100-Media $\left\{\right.$ MSSE < MSE $\left.{ }^{*}\right\}$ \\
\hline
\end{tabular}
cero, supone una predicción perfecta, lo cual es muy difícil que suceda.

Figura 3. Medidas de selección, precisión e Interpretación para métodos de pronósticos.

\section{RESULTADOS}

Los resultados presentados se resumen en las siguientes categorías:

$\checkmark$ Análisis del comportamiento histórico de los eventos relacionados a la seguridad y salud en el trabajo.

$\checkmark$ Determinación mediante la técnica de Pareto de los rubros con mayor generación de costos en S\&SO.

$\checkmark$ Pronóstico para el año 2014 de los costos mencionados en el ítem anterior.

$\checkmark$ Proyección del Estado de Resultados para el año 2014 en ausencia del SGSST (las proyecciones se realizaron con el SPSS 20, con el modelador experto del mismo).

$\checkmark$ Determinación del criterio para la reducción de costos de la compañía asociados al S\&SO.

$\checkmark$ Proyección del Estado de Resultados para el año 2014 considerando una reducción de los costos relacionados con el SGSST con base en el criterio establecido por la empresa.

A. Comportamiento Siniestralidad en los últimos años

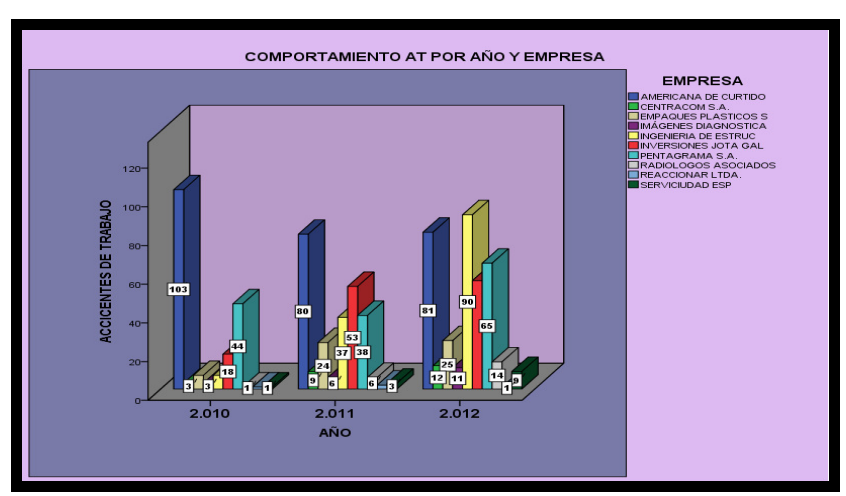

Figura 5. Descriptivo para las empresas en los últimos años (americana de azul) 
En la figura 5, arriba presentada, se describe mediante la técnica de Pareto la priorización en el ámbito de la seguridad y salud en el trabajo, en resumen los incidentes, accidentes, enfermedades, incapacidades, indemnizaciones y demás que generan retrasos en los procesos, afectan la programación de la producción, el cumplimiento de compromisos con los proveedores y clientes y en términos generales afectan la rentabilidad de la empresa; es así que la empresa Americana de Curtidos Ltda. \&Cía. S.C.A viene presentando en los últimos años un creciente comportamiento en los accidentes de trabajo (AT).

B. Identificación de los conceptos que mayor generan costos por la ausencia de la implementación del sistema de gestión en S\&SO Oshas 18001:2007 hasta el 2013. Aplicación de la técnica de Pareto $(80$ - 20)

\begin{tabular}{|c|c|c|c|c|}
\hline$\#$ & CONCPETO & VALOR & PORCENTAIE & PORCENTAJE ACUMULADO \\
\hline 1 & $\begin{array}{c}\text { Costo de los sueldos ppagados } \\
\text { a supervisores por el tiempo } \\
\text { requerido para actividadas } \\
\text { necessrias debidas al } \\
\text { accidente }\end{array}$ & $23,992,787,50$ & $18,05 \%$ & $18,05 \%$ \\
\hline 2 & $\begin{array}{c}\text { Costos asumidos por el pago } \\
\text { de personal en el reemplazo } \\
\text { de trabajadores } \\
\text { incapacitdodos por } \\
\text { incapacidades. }\end{array}$ & $21,307,822,43$ & $16,03 \%$ & $34,08 \%$ \\
\hline 3 & $\begin{array}{c}\text { Costos asumidos por el } \\
\text { tiempo de la investigadón } \\
\text { de los Accidentes Internos, } \\
\text { en donde participan los } \\
\text { supervisores ytrabajadores } \\
\text { miembros del COPASST. }\end{array}$ & $21.284,234,05$ & $16,01 \%$ & $50,09 \%$ \\
\hline 4 & $\begin{array}{c}\text { Costos pagados por la } \\
\text { investigación de A.T "graves" } \\
\text { por Profesionales en Salud } \\
\text { Ocupacionales externos. }\end{array}$ & $20.419 .640,72$ & $15,36 \%$ & $65,45 \%$ \\
\hline 5 & \begin{tabular}{|c|} 
Costo en salarios caussado \\
por la redución en \\
producción de trabajadoess \\
lesionados después de su \\
regreso a t trabajo
\end{tabular} & $19,051.863,13$ & $14,33 \%$ & $79,78 \%$ \\
\hline
\end{tabular}

\begin{tabular}{|c|c|c|c|c|}
\hline $6 \mathrm{pa}$ & $\begin{array}{c}\text { Costo de los salarios } \\
\text { pagados por tiempo perdido } \\
\text { al trabajajdor lesionado }\end{array}$ & $3.560 .274,93$ & $2,68 \%$ & $92,96 \%$ \\
\hline 7 & \begin{tabular}{|c|} 
Costo de evaluaciones \\
ambientales (Sonometrias, \\
dosimetrias, confort \\
térmico, luxometrias, \\
medición de gases, \\
medición de vibraciones)
\end{tabular} & $3.286 .310,75$ & $2,47 \%$ & $82,25 \%$ \\
\hline 8 & $\begin{array}{l}\text { Costo Programa Salud } \\
\text { Mental-SVVERiesgo } \\
\text { Psicosocial }\end{array}$ & $3.190,395,88$ & $2,40 \%$ & $84,65 \%$ \\
\hline$g^{D}$ & $\begin{array}{c}\text { Dotación de las brigadas de } \\
\text { emergencias }\end{array}$ & $2.845 .845,13$ & $2,14 \%$ & $86,79 \%$ \\
\hline $10^{c}$ & $\begin{array}{c}\text { Capacitación en Seguridady } \\
\text { Salud en el Trabajo }\end{array}$ & $2.570 .500,63$ & $1,93 \%$ & $88,73 \%$ \\
\hline 11 & $\begin{array}{c}\text { Costo neto necesario para } \\
\text { reparar, remplazary } \\
\text { ordenar los materialesy } \\
\text { equipos que resultaron } \\
\text { dañados en accidente }\end{array}$ & $2.249,107,00$ & $1,69 \%$ & $94,65 \%$ \\
\hline 12 & $\begin{array}{c}\text { Costos EPl e Implementos } \\
\text { de Seguridad } \\
\text { Industrial|(Creciente) }\end{array}$ & $20.068 .357,50$ & $1,56 \%$ & $90,28 \%$ \\
\hline 13 & \begin{tabular}{|c|}
$\begin{array}{c}\text { Costo servicios médicos } \\
\text { salud ocupacional } \\
\text { (Profesional médico que } \\
\text { realiza las valoraciones) }\end{array}$ \\
\end{tabular} & $2.013 .439,50$ & $1,51 \%$ & $96,17 \%$ \\
\hline 14 & $\begin{array}{c}\text { Valor exámenes médicos } \\
\text { ocupacionales (Ingreso, } \\
\text { periódicos, retiro) }\end{array}$ & $1.652 .260,75$ & $1,24 \%$ & $97,41 \%$ \\
\hline 15 & $\begin{array}{c}\begin{array}{c}\text { Costo señalización seguridad } \\
\text { industrial }\end{array} \\
\end{array}$ & $1.579 .189,75$ & $1,19 \%$ & $98,60 \%$ \\
\hline 16 & $\begin{array}{c}\text { Costo adicional por trabajo } \\
\text { en tiempo extraordinario } \\
\text { debido al accidente. }\end{array}$ & $1.187 .417,13$ & $0,89 \%$ & $99,49 \%$ \\
\hline 17 & $\begin{array}{l}\text { Costo médico no asegurado, } \\
\text { cubierto por la empresa }\end{array}$ & $526.568,38$ & $0,40 \%$ & $99,89 \%$ \\
\hline 18 & \begin{tabular}{|c|} 
Costo del aprendizaje de \\
nuevoso trabajajdores. \\
Cuando una lesion resulta \\
tan seria que debe de \\
contratarse a un nuevo \\
trrabajador otransferir a \\
otro ocasiona un nuevo \\
costo \\
\end{tabular} & $150.112,25$ & $0,11 \%$ & $100,00 \%$ \\
\hline & TOTAL & $132.936 .127,37$ & $100 \%$ & \\
\hline
\end{tabular}

De los resultados generados en la tabla anterior y mediante la técnica de Pareto, los 5 conceptos identificados, en orden de peso son:

1. Costo de los sueldos pagados a supervisores por el tiempo requerido para actividades necesarias debidas al accidente.

2. Costos asumidos por el pago de personal en el reemplazo de trabajadores incapacitados por incapacidades.

3. Costos asumidos por el tiempo de la investigación de los Accidentes Internos, en donde participan los supervisores y trabajadores miembros del COPASST. 
4. Costos pagados por la investigación de A.T "graves" por Profesionales en Salud Ocupacionales externos.

5. Costo en salarios causado por la reducción en producción de trabajadoreslesionados después de su regreso al trabajo.

En la figura 4, que aparece en la siguiente columna se presenta el gráfico tipo Pareto, que permite identificar los aspectos de mayor peso en el análisis financiero.

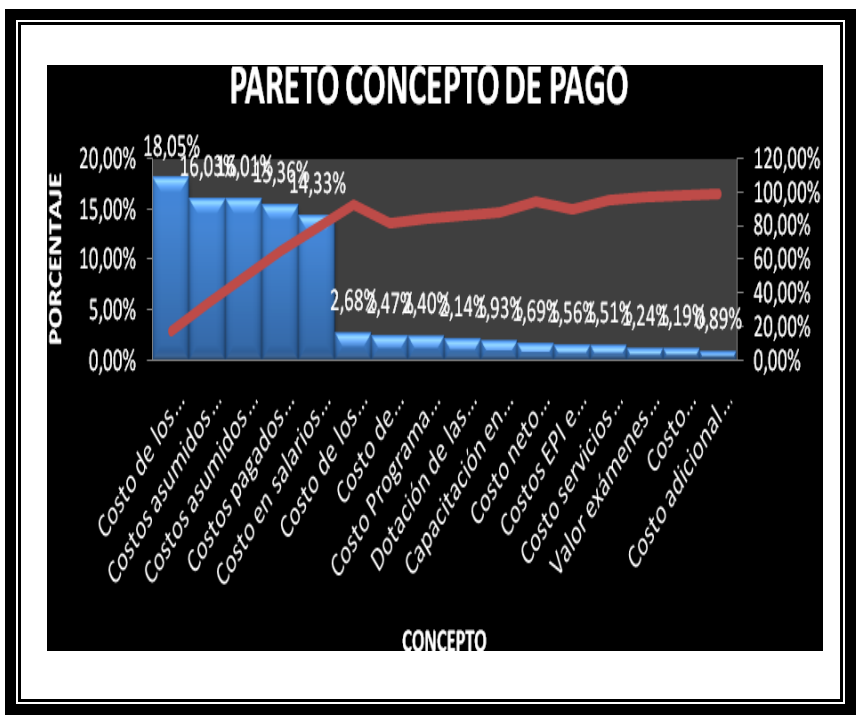

Figura 4. Gráfico de Pareto.

F. Comportamiento hasta el 2013 y proyección al 2014 de dos de los cinco conceptos de mayor peso en los costos asociados a gastos en seguridad ysalud en el trabajo.

1. Costo de los sueldos pagados a supervisores por el tiempo requerido para actividades necesarias debidas al accidente.

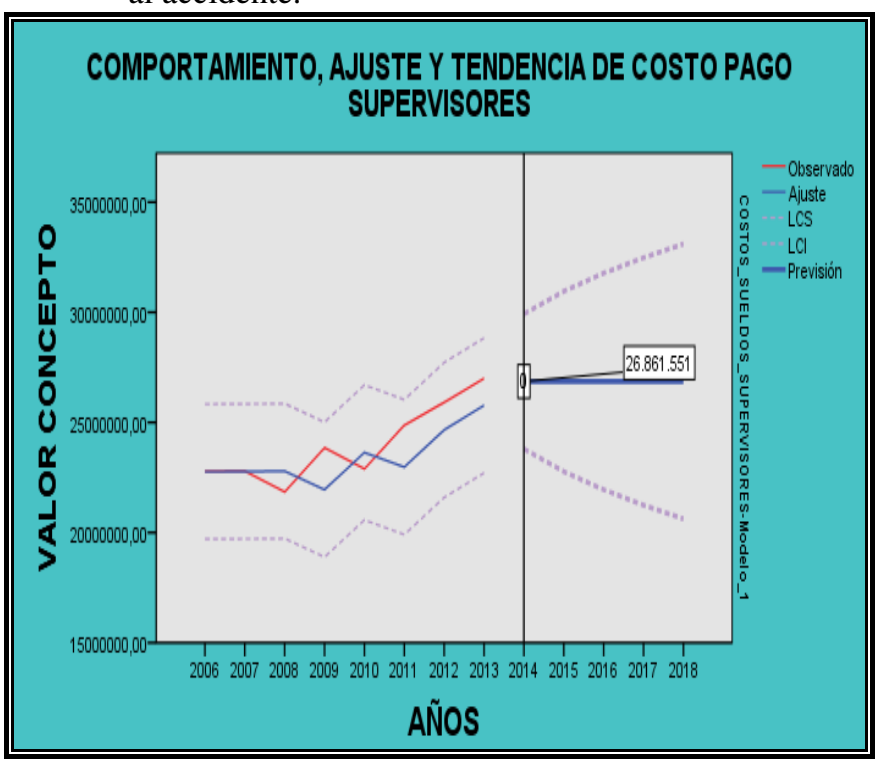

Figura 5. Proyección costo pagado a supervisores
En la grafica anterior se puede ver que en ausencia del SGSST, se estima que el pago por este concepto para el 2014 es de \$26.661.551.

2. Costos asumidos por el pago de personal en el reemplazo de trabajadores incapacitados por incapacidades

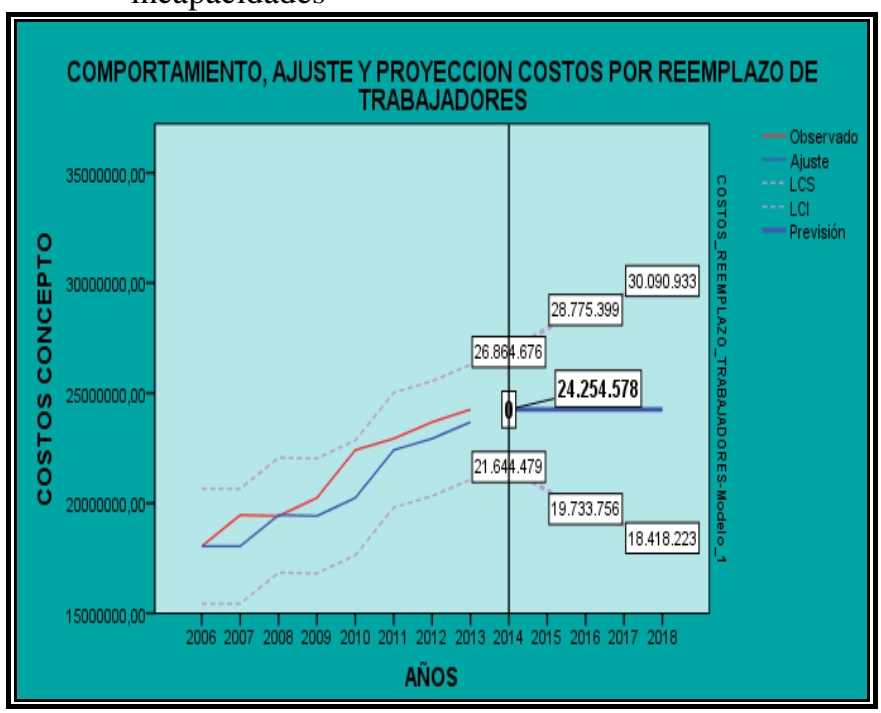

Figura 6. Proyección costo pagado por reemplazo de trabajadores.

De la gráfica anterior se puede ver que en ausencia del SGSST, se estima que el pago por este concepto para el 2014 es de \$24.254.568, con un error de estimación de \$2.610.098, generando así un límite superior de estimación de $\$ 26.864 .676$ y un límite inferior para esta estimación de \$21.644.479.

El mismo análisis se hizo con los otros tres factores identificados con la técnica de Pareto. Estas proyecciones se hicieron haciendo uso del Modulo Modelador Experto del programa SPSS versión 20.

G. Proyección del Estado de Resultados para el año 2014 en ausencia del SGSST

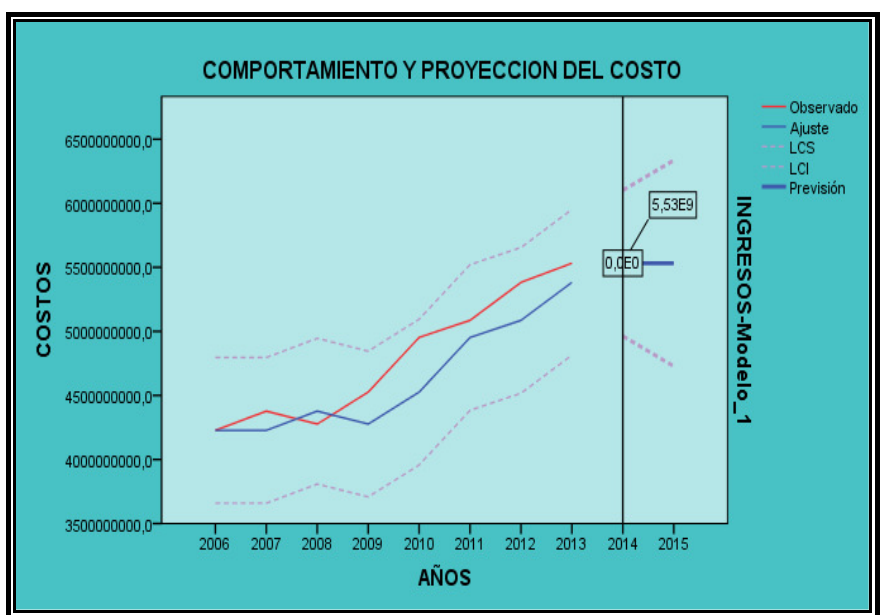

Figura 7. Proyección de los ingresos 
Para el 2014 se aprecia la cifra esperada para el año 2014 la cual es de \$5.532.040.914,4 (sin la implementación de un SGSST). En la figura 8 se leen los estadísticos que arrojó el modelo de pronósticos para el ingreso en el programa SPSS. Efectivamente, es el mejor modelo de todos, pues el BIC (Bayesian Information Criterion) es muy pequeño (38.856) lo que indica una muy buena medida de bondad de ajuste del modelo de datos.

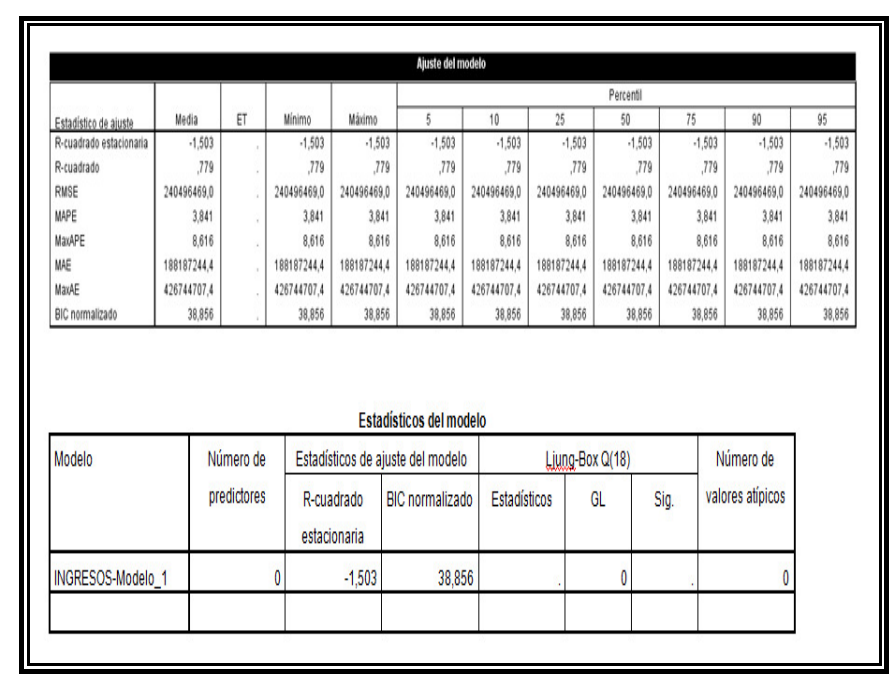

Figura 8. Resumen estadísticosproyección ingresos

Para la proyección del patrimonio, los costos de producción y costos de ventas y gastos se aplicó la misma metodología.

La figura 9 detalla entonces el Estado de Resultados proyectado para el año 2014 en ausencia del SGSST:

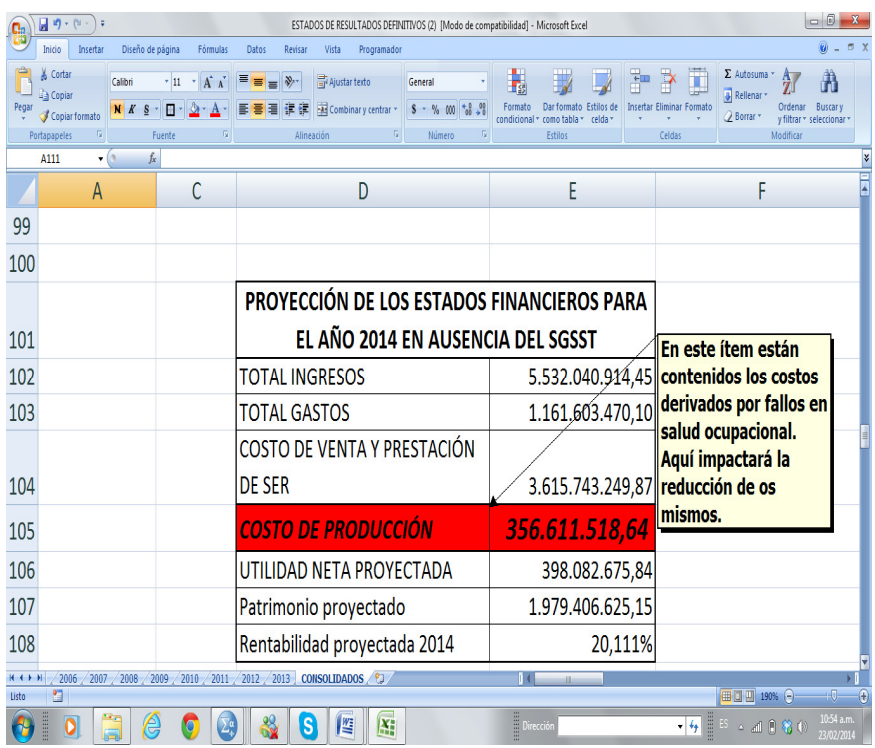

Figura 9. Proyección de los Estados Financieros para el año 2014 en ausencia de un SGSST.
H. Determinación del criterio para la reducción de costos de la compañía asociados al S\&SO.

Para fijar una política de reducción de costos en relación con el S\&SO, la empresa se valió de dos herramientas: la tasa de accidentalidad y la matriz de identificación de peligros.

\section{Tasa de Accidentalidad}

Se promedió esta tasa con información histórica arrojando un resultado de un 27,25\%, como lo indica la figura 10.

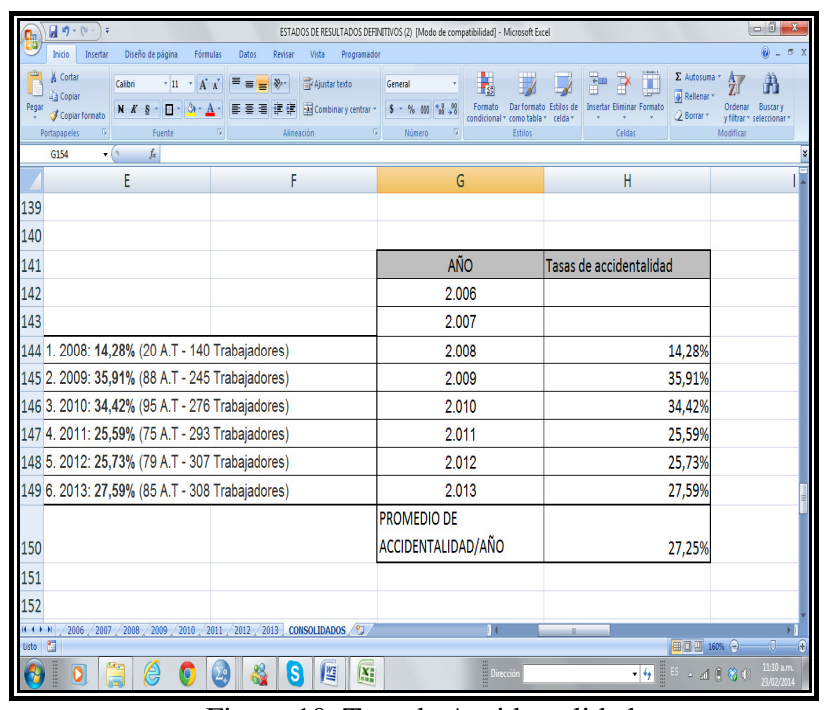

Figura 10. Tasa de Accidentalidad

\section{Matriz de Identificación de Peligros [7]}

Esta matriz permite identificar riesgos en las diferentes áreas de la compañía.El análisis de esta, consiste en que se eliminaron las áreas o secciones de trabajo que en su valoración del "riesgo" no dieron como resultado "No Aceptable", o sea dieron como resultado "Aceptable"; lo cual indica que los controles en estas secciones están siendo efectivos y no representan un sobre-costo en las inversiones financieras que realiza la empresa. Por otro lado en el análisis final quedaron la mayoría, a las cuales también se le eliminaron los peligros identificados que no dieron en su valoración "No Aceptable", siendo estos procesos, actividades y tareas las más representativas, y lógicamente centrando la atención en las secciones que históricamente y estadísticamente son las más accidentadas de la empresa, siendo estas (secciones) las que soportan y justifican el análisis Pareto de las variables que más inciden directamente sobre los costos, ya que allí se ven reflejadas directamente. Importante referir que dentro de la metodología utilizada (GTC 45:2010-2012), la empresa incluyo una segunda valoración en donde se puede inferir lo siguiente: 
1. Valoración sin control y valoración con los controles implementados.

2. Con la implementación del SG-SST basado en la NTC-OHSAS 18001:2007 se espera que no solo se vea reflejado en los comportamientos financieros, sino que además en la segunda valoración el Riesgo, este "ACEPTABLE", (El cual es un riesgo que ha sido reducido a un nivel que la organización puede tolerar, respecto a sus obligaciones legales y su propia política en seguridad y salud en el trabajo).

A partir de la tasa promedio de accidentalidad (27.25\%) y el análisis en campo para la actualización de la matrizdeidentificación de peligros, la empresa determina como política empresarial que bajo la implementación del Sistema de Gestión de Seguridad y Salud en el Trabajo, este DEBE IMPACTAR reduciendo en al menos un 20\% los costos de salud ocupacional y costos de producción.

I. Proyección del Estado de Resultados para el año 2014 considerando una reducción de los costos relacionados con el SGSST con base en el criterio establecido por la empresa.

Con la política de reducción de costos en S\&SO fijada por la compañía, se procedió a presupuestar los costos para el año 2014 con ese decremento de tal manera que se obtuvo un presupuesto de Estado de Resultados para el año 2014 como lo indica la figura 11:

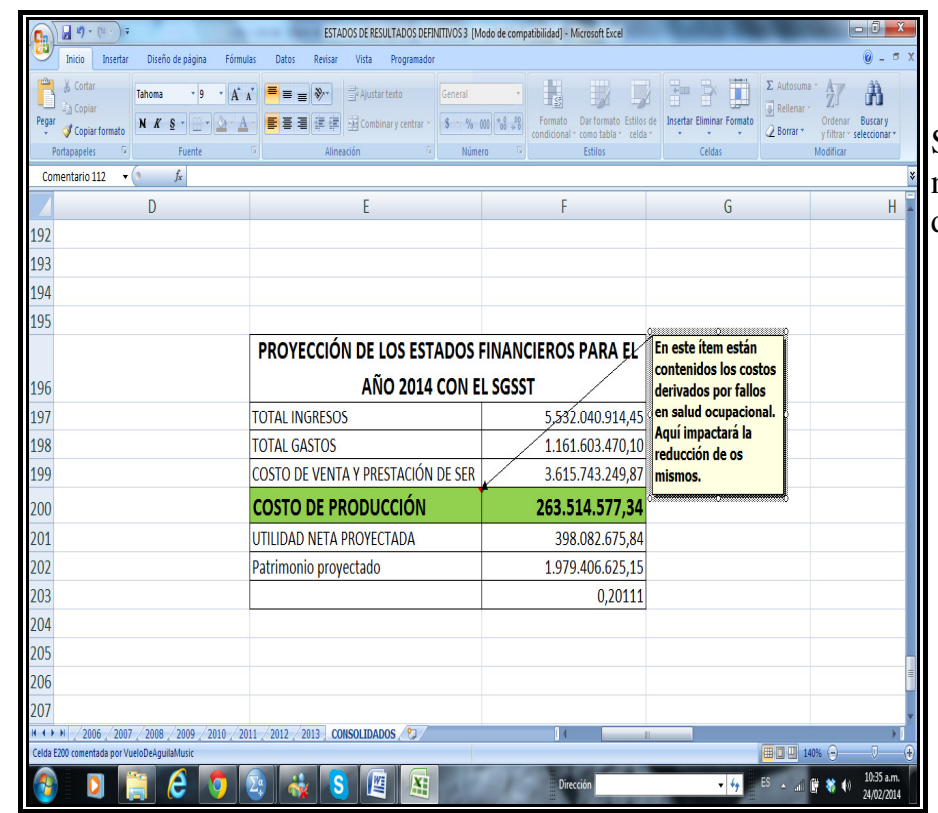

Figura 11. Proyección de Estados de Resultados para el año 2014 con SGSST

Una reducción del 20\% implica que para el año 2014 la tasa esperada de 0,2759 se reduciría a:
$(27,59 \%) *(0,20)=5,52 \%$, entonces $27,59 \%-5,52 \%=$ $22,07 \%$ que sería la nueva tasa de accidentalidad para el año 2014.

Es decir, la proporción de costos de no calidad en salud ocupacional con esta tasa traducida en pesos sería como sigue:

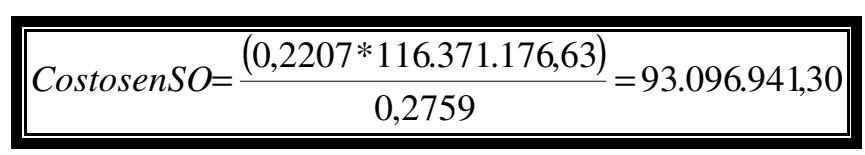

$\operatorname{Re}$ ducciónDeCostos $2014=\frac{(93.096 .941,30-116.371 .176 .63)}{116.371 .176,63}=-0,20$

Lo cual indica que con la implementación del SGSST, se pasará este año de \$11.371.176,63 sin SGSST a $\$ 93.096 .941,3$, con SGSST, es decir una reducción de $\$ 23.274 .235,32$, que corresponde a una reducción del $20 \%$ por concepto de costos en Salud Ocupacional.

Esta reducción de costos en salud ocupacional, impactan directamente los costos de producción de la empresa. Es decir, que si el presupuesto para el 2014 en materia de costos de producción (incluyendo los costos de salud ocupacional con la tasa esperada de accidentalidad del 27,59\%) era de $356.611 .518,54$, ahora la nueva cifra para el 2014 resultante de disminución de costos en SO (-20\%) es de:

$356.611 .518,54-93.096 .941,30=263.514 .577 .34$

El rubro de costos de producción sufriría una variación como sigue:

$\operatorname{VarCPs} 2014=\frac{(263.514 .577,34-356.611 .518,64)}{356.611 .518,64}=-0,2611$

Si en el artículo se utilizan ecuaciones, estas deberán tener numeración consecutiva, así no las cite o use en el texto. Se debe definir su procedencia.

\section{CONCLUSIONES}

1. Los cincos aspectos más importantes relacionados con gastos generados en el área de Seguridad y Salud en el Trabajo en la EMPRESA AMERICANA DE CURTIDOS LTDA. \& CIA. S.C.A identificados mediante la Técnica de Pareto a los Estados de Resultados de los años 2006 al 2013 son:

$>$ Costo de los sueldos pagados a supervisores por el tiempo requerido para actividades necesarias debidas al accidente.

$>$ Costos asumidos por el pago de personal en el reemplazo de trabajadores incapacitados por incapacidades.

$>$ Costos asumidos por el tiempo de la investigación de los Accidentes Internos, en donde participan los supervisores y trabajadores miembros del COPASST.

> Costos pagados por la investigación de A.T "graves" por Profesionales en Salud Ocupacionales externos. 
$>$ Costo en salarios causado por la reducción en producción de trabajadores lesionados después de su regreso al trabajo.

2. El diligenciamiento y análisis de la matriz de Peligros, así como de el comportamiento de la tasa de accidentalidad de los últimos años permitió conocer el promedio de esta, valor que fue de:

Tasa promedio de accidentalidad $=27.25 \%$

3. A partir de la tasa promedio de accidentalidad (27.25\%) y el análisis en campo para la actualización de la matriz de identificación de peligros, la empresa determina como política empresarial que bajo la implementación del Sistema de Gestión de Seguridad y Salud en el Trabajo, este DEBE IMPACTAR reduciendo en al menos un $20 \%$ los costos de salud ocupacional y costos de producción.

4. Con la política de reducción de costos en S\&SO fijada por la compañía, se procedió a presupuestar los costos para el año 2014 con ese decremento de tal manera que se obtuvo un presupuesto de Estado de Resultados para el año 2014, Lo cual indica que con la implementación del SGSST, se pasará este año de $\$ 11.371 .176,63$ sin SGSST a \$93.096.941,3, con SGSST, es decir una reducción de \$23.274.235,32, que corresponde a una reducción del $20 \%$ por concepto de costos en Salud Ocupacional.

\section{RECOMENDACIONES}

$>$ El modelo representa una primera aproximación para el análisis de costos de no calidad utilizando el modelador experto SPSS. Esta técnica se constituye en una base sobre la cual posteriores estudios podrán añadir valor.

> Para toda empresa es de vital importancia el registro de información de todos sus aspectos de operación, pues esto facilita el análisis y establecimiento de relaciones entre las diferentes áreas de esta, así como el impacto o relación de causalidad que se puedan establecer para optimizar recursos, tiempo, personal y dinero.

$>$ La implementación de los Sistemas de Gestión de Calidad tienen además como objetivo impactar positivamente el desempeño financiero de las organizaciones. La ventaja competitiva implica que éstas puedan ser más rentables frente a otras empresas del mismo sector. Por ello debe incluirse un proceso financiero que esté en la capacidad de generar y procesar la información necesaria que permita, en el marco de las Norma de Calidad en integración especial con la OSHAS 18001:2007 y otra normas mejorar los indicadores de rentabilidad.

> El análisis realizado en la presente investigación sugiere realizar no solo en el ámbito de Seguridad y Salud en el Trabajo, sino también por lo menos en Producción (Control Total de la Calidad), y en medio ambiente, con lo cual se espera que los resultados encontrados IMPACTEN mucho más en la rentabilidad de la empresa.

\section{REFERENCIAS}

ESTADOS FINANCIEROS. Recuperado septiembre de 2013. http://www.monografias.com/trabajos5/estafinan/esta finan.shtml

RENTABILIDAD FINANCIERA. Monografía. Recuperado enero de 2014. http://www.slideshare.net/jers1504/rentabilidadfinanciera-roe

[3] COSTOS DE PRODUCCIÓN. Monografía. Recuperado febrero de 2014. http://www.fao.org/docrep/003/v8490s/v8490s06.htm

DEFINICION Y APLICACIONES DE LA TECNICA DIAGRAMA DE PARETO. Articulo. Recuperado septiembre de 2013.

http://www.fundibeq.org/opencms/export/sites/defaul t/PWF/downloads/gallery/methodology/tools/diagra ma_de_pareto.pdf

MÉTODOS DE SUAVIZAMIENTO Y PRONÓSTICO PARA SERIES DE TIEMPO.

Recuperado. Agosto de 2013. http://www.monografias.com/trabajos $87 /$ metodossuavizamiento-y-pronostico-series-tiempo/metodossuavizamiento-y-pronostico-seriestiempo.shtml\#ixzz2uLcRFRBO

¿Cómo MEDIR LA PRECISION Y EFECTIVIDAD DE LOS PRONOSTICOS? Articulo. Recuperado enero de 2014.

http://www.slideshare.net/tomgalvez/mtodos-paramedir-la-precisin.

[7] MATRIZ DE FACTOR DE RIESGOS. PDF. Recuperado febrero de 2014. http://www.google.com.co/?gfe_rd=ctrl\&ei=q7gMU 7TOD67Q8gePu4CgDQ\&gws rd=cr\#q=matriz + de+ peligros + gtc +45 